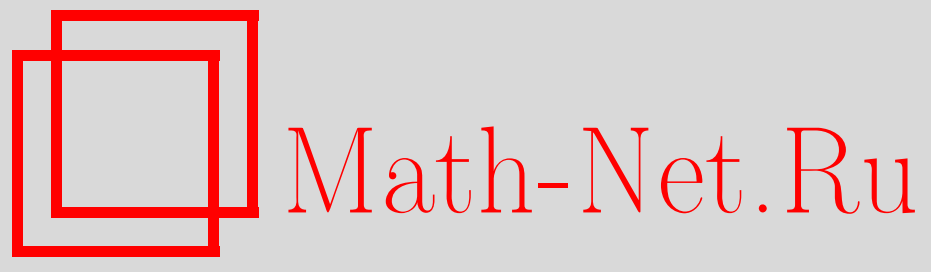

А. П. Шашкин, Сильная гауссовская аппроксимация ассоциированного случайного поля, УМН, 2007, том 62, выпуск 5, 171-172

DOI: https://doi.org/10.4213/rm7331

Использование Общероссийского математического портала Math-Net.Ru подразумевает, что вы прочитали и согласны с пользовательским соглашением http://www . mathnet.ru/rus/agreement

Параметры загрузки:

IP : 3.85 .73 .92

26 апреля 2023 г., 11:35:40

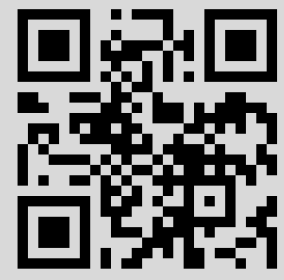




\section{Сильная гауссовская аппроксимация ассоциированного случайного поля}

\section{А. П. Шашкин}

Заметка посвящена установлению сильного принципа инвариантности для ассоциированного случайного поля. Случайное поле $X=\left\{X_{j}, j \in \mathbb{Z}^{d}\right\}$ называется ассоциированным [1], если $\operatorname{cov}\left(f\left(X_{i_{1}}, \ldots, X_{i_{n}}\right), g\left(X_{i_{1}}, \ldots, X_{i_{n}}\right)\right) \geqslant 0$ для любого $n \in \mathbb{N}$, произвольного набора попарно различных точек $\left\{i_{1}, \ldots, i_{n}\right\} \subset \mathbb{Z}^{d}$ и любых ограниченных покоординатно неубывающих борелевских функций $f, g: \mathbb{R}^{n} \rightarrow \mathbb{R}$. Ассоциированные случайные поля встречаются во многих задачах статистической физики, теории надежности, статистики, теории случайных мер. Известны условия ассоциированности процессов авторегрессии, полей дробового шума, кластерных случайных мер, обобщений модели Изинга. За последние 30 лет для ассоциированных случайных полей получены такие классические предельные теоремы теории вероятностей, как центральная предельная теорема с оценками скорости сходимости, законы больших чисел, законы повторного логарифма и др. (см. [2]). При изучении таких полей очень важен коэффициент Кокса-Гримметта

$$
u_{r}=\sup _{j \in \mathbb{Z}^{d}} \sum_{k \in \mathbb{Z}^{d}:|k-j| \geqslant r} \operatorname{cov}\left(X_{j}, X_{k}\right), \quad r \in \mathbb{N} .
$$

Здесь $|j|=\max _{i=1, \ldots, d}\left|j_{i}\right|$ для $j \in \mathbb{Z}^{d}$. Сильные принципы инвариантности для ассоциированных процессов и полей были получены в работах [3] и [4] соответственно. В обеих работах от коэффициента Кокса-Гримметта требовалось экспоненциальное убывание $u_{r}$ на бесконечности. В данной заметке мы предположим лишь степенной характер убывания. Напомним, что сильный принцип инвариантности был впервые получен В. Штрассеном для независимых слагаемых и является очень мощным утверждением, из которого вытекает, в частности, закон повторного логарифма.

Для $n \in \mathbb{N}^{d}$ обозначим $\langle n\rangle=n_{1} \ldots n_{d}$ и $n-1=\left(n_{1}-1, \ldots, n_{d}-1\right)$. Запись $m \leqslant n$ $\left(m, n \in \mathbb{Z}^{d}\right)$ понимается в смысле $m_{i} \leqslant n_{i}, i=1, \ldots, d$. Прямоугольником $(a, b]$, где $a, b \in \mathbb{Z}^{d}$ и $a \leqslant b$, назовем множество $\left(\left(a_{1}, b_{1}\right] \times \cdots \times\left(a_{d}, b_{d}\right]\right) \cap \mathbb{Z}^{d}$. Для $\tau>0$ положим $G_{\tau}=\left\{n \in \mathbb{N}^{d}: n_{k} \geqslant\left(\prod_{j \neq k} n_{j}\right)^{\tau}, k=1, \ldots, d\right\}$. Для конечного множества $V \subset \mathbb{Z}^{d}$ положим $S(V)=\sum_{j \in V} X_{j}$, и, наконец, для $n \in \mathbb{N}^{d}$ пусть $S_{n}=S((0, n])$. Символ Law (·) далее означает распределение случайного элемента.

Теорема. Пусть стационарное в широком смысле центрированное случайное поле $X$ ассочиированно $и \sup _{j \in \mathbb{Z}^{d}} \mathrm{E}\left|X_{j}\right|^{2+\delta}<\infty$ для некоторого $\delta>0$. Предположим, что для некоторого $\lambda>0$ справедливо соотношение $u_{r}=O\left(r^{-\lambda}\right)$ nри $r \rightarrow \infty$, где $u_{r}$ определено в (1). Тогда для каждого $\tau>0$ можно переопределить поле $X$ на новом вероятностном пространстве вместе с d-параметрическим броуновским движением $\left\{W_{t}, t \in \mathbb{R}_{+}^{d}\right\}$, причем существует такое $\varepsilon>0$, что с вероятностью единица

$$
\langle n\rangle^{\varepsilon-1 / 2}\left(S_{n}-\sigma W_{n}\right) \rightarrow 0 \quad \text { npu } n \rightarrow \infty, n \in G_{\tau},
$$

где $\sigma^{2}=\sum_{j \in \mathbb{Z}^{d}} \operatorname{cov}\left(X_{0}, X_{j}\right)$, а записъ $n \rightarrow \infty$ означает, что $n_{i} \rightarrow \infty, i=1, \ldots, d$.

Основную роль в доказательстве играют две леммы.

Лемма 1. Пусть $Y=\left(Y_{1}, \ldots, Y_{m}\right)$ - ассоциированный случайный вектор, и пусть $\mathrm{E} Y_{j}^{2}<\infty, j=1, \ldots, m$. Предположим, что случайная величина $Z$ не зависит от

Работа выполнена при поддержке РФФИ (гранты № 05-01-00944 и № 07-01-00373). 
вектора $\left(Y_{1}, \ldots, Y_{m-1}\right)$ u $\operatorname{Law}(Z)=\operatorname{Law}\left(Y_{m}\right)$. Тогда для любой функции $f: \mathbb{R}^{m} \rightarrow \mathbb{R}$, имеющей ограниченные частные производные второго порядка, верна оченка

$$
\left|\mathrm{E} f\left(Y_{1}, \ldots, Y_{m-1}, Y_{m}\right)-\mathrm{E} f\left(Y_{1}, \ldots, Y_{m-1}, Z\right)\right| \leqslant \sum_{k=1}^{m-1}\left\|\frac{\partial^{2} f}{\partial x_{k} \partial x_{m}}\right\|_{\infty} \operatorname{cov}\left(Y_{k}, Y_{m}\right),
$$

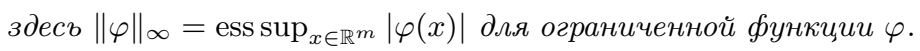

Лемма 2. Пусть $Y=\left(Y_{1}, \ldots, Y_{m}\right)$ - тот же случайный вектор, что в лемме 1. Тогда $Y$ можно переопределить на веролтностном пространстве, на котором заданы независимые случайные величины $Z_{1}, \ldots, Z_{m}$ такие, что $\operatorname{Law}\left(Z_{k}\right)=\operatorname{Law}\left(Y_{k}\right)$ $u \mathrm{P}\left(\left|Y_{k}-Z_{k}\right|>a_{k}\right) \leqslant a_{k}$ для $k=1, \ldots, m$, где $a_{k}=A \sum_{v=k}^{m} v^{1 / 3}\left(\sum_{j=1}^{v-1} \operatorname{cov}\left(Y_{j}, Y_{v}\right)\right)^{1 / 3}$ и $A>0$ есть абсолютная константа.

ДокАЗАТЕЛЬСтво леммы 1 основано на теореме о функциях от ассоциированных векторов [5]. Лемма 2 следует из леммы 1, неравенства Сенатова для метрики Леви-Прохорова [6], и теоремы Штрассена об этой метрике [7].

Дальнейшее доказательство теоремы следует в основном пути работ [4] и [8]. Зададим числа $\alpha \in \mathbb{N}, \beta \in(0, \alpha)$. Пусть $\left\{w_{k}, k \in \mathbb{N}^{d}\right\}$ - независимые друг от друга и от $X$ случайные величины, причем $w_{k} \sim N\left(0,\langle k\rangle^{\beta}\right)$. Для $k \in \mathbb{Z}_{+}^{d}$ введем точку $\mathrm{N}_{k} \in \mathbb{N}^{d}$, $i$-я координата которой равна $\sum_{l=0}^{k_{i}} l^{\alpha}$, и положим $B_{k}=\left(\mathrm{N}_{k-1}, \mathrm{~N}_{k}\right], k \in \mathbb{N}^{d}$. Пусть дано $n \in G_{\tau}$. Найдем $k \in \mathbb{N}^{d}$, для которого $n \in B_{k}$. Тогда $S_{\mathrm{N}_{k}}$ равно

$$
\sum_{t \leqslant k} S\left(B_{k}\right)=\sum_{t \leqslant k} \sqrt{\sigma_{t}^{2}+\tau_{t}^{2}}\left(\xi_{t}-\eta_{t}\right)+\sum_{t \leqslant k}\left(\sqrt{\frac{\sigma_{t}^{2}+\tau_{t}^{2}}{\left|B_{t}\right|}}-\sigma\right) \sqrt{\left|B_{t}\right|} \eta_{t}-\sum_{t \leqslant k} w_{k}+\sigma \sum_{t \leqslant k} \sqrt{\left|B_{t}\right|} \eta_{t},
$$

где $\sigma_{t}^{2}=\mathrm{D} S\left(B_{t}\right), \tau_{t}=\mathrm{D} w_{t}, \quad \xi_{t}=\left(\sigma_{t}^{2}+\tau_{t}^{2}\right)^{-1 / 2}\left(S\left(B_{t}\right)+w_{t}\right), \quad \eta_{t}=\Phi^{-1}\left(F_{t}\left(\xi_{t}\right)\right)$, a $F_{t}(x)=\mathrm{P}\left(\xi_{t} \leqslant x\right)$ и $\Phi$ есть функция распределения стандартного нормального закона. Основной вклад в выражение (2) вносит последнее слагаемое, и для его аппроксимации броуновским движением к случайным величинам $\left(\eta_{m}\right)_{m \in \mathbb{N}}$ можно применить лемму 2. Прочие слагаемые, а также разность $S_{n}-S_{\mathrm{N}_{k}}$ оцениваются (при должном выборе $\alpha$ и $\beta$ ) аналогично [4] и [8]. Отметим, что в работе [8] используется экспоненциальное убывание более общего коэффициента зависимости, чем $u_{r}$.

Автор признателен А. В. Булинскому и В. В. Сенатову за полезные обсуждения.

\section{Список литературы}

[1] J.D. Esary, F. Proschan, D.W. Walkup, Ann. Math. Statist., 38 (1967), 1466-1474. [2] A. Bulinski, A. Shashkin, Limit theorems for associated random fields and related systems, World Sci. Publ., Singapore, 2007. [3] H. Yu, Ann. Probab., 24:4 (1996), 2079-2097. [4] R. M. Balan, Ann. Probab., 33:2 (2005), 823-840. [5] T. C. Christofides, E. Vaggelatou, J. Multivariate Anal., 88:1 (2004), 138-151. [6] В. В. Сенатов, Теория вероятн. и ее примен., 29:1 (1984), 108-113. [7] V. Strassen, Ann. Math. Statist., 36 (1965), 423-439. [8] A. V. Bulinski, A. P. Shashkin, Dynamics and Stochastics. Festschrift in honor of M. S. Keane (Eindhoven, The Netherlands, 2005), IMS Lecture Notes Monogr. Ser., 48, IMS, Beachwood, 2006, 128-143.

А. П. Шашкин (А.P. Shashkin)

Московский государственный университет им. М. В. Ломоносова

E-mail: shashkin@mech.math.msu.su
Представлено А. В. Булинским Принято редколлегией 20.07.2007 